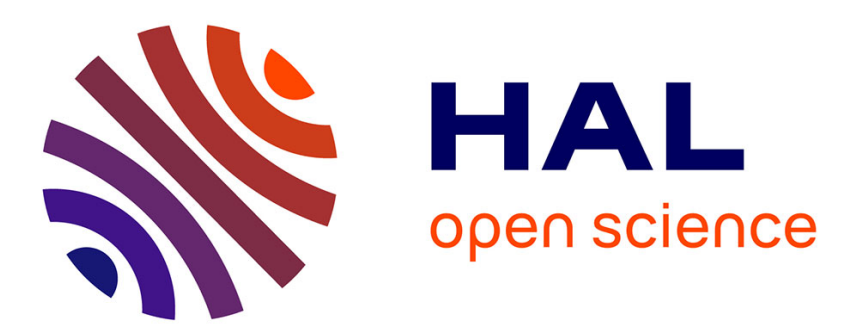

\title{
An analytical solution for an inflated orthotropic membrane tube with an arbitrarily oriented orthotropy basis
}

Quang Tung Nguyen, Jean-Christophe Thomas, Anh Le Van

\section{- To cite this version:}

Quang Tung Nguyen, Jean-Christophe Thomas, Anh Le Van. An analytical solution for an inflated orthotropic membrane tube with an arbitrarily oriented orthotropy basis. Engineering Structures, 2013, 56, pp.1080-1091. 10.1016/j.engstruct.2013.06.012 . hal-01004981

\section{HAL Id: hal-01004981 \\ https://hal.science/hal-01004981}

Submitted on 28 Oct 2016

HAL is a multi-disciplinary open access archive for the deposit and dissemination of scientific research documents, whether they are published or not. The documents may come from teaching and research institutions in France or abroad, or from public or private research centers.
L'archive ouverte pluridisciplinaire HAL, est destinée au dépôt et à la diffusion de documents scientifiques de niveau recherche, publiés ou non, émanant des établissements d'enseignement et de recherche français ou étrangers, des laboratoires publics ou privés.

\section{(c)(1)}

Distributed under a Creative Commons Attribution| 4.0 International License 


\title{
An analytical solution for an inflated orthotropic membrane tube with an arbitrarily oriented orthotropy basis
}

\author{
Quang Tung Nguyen, Jean-Christophe Thomas, Anh Le van \\ LUNAM Université, Université de Nantes-Ecole Centrale Nantes, GeM (Institute for Research in Civil and Mechanical Engineering), CNRS UMR 6183, 2, rue de la Houssinière, \\ BP 92208, 44322 Nantes Cedex 3, France
}

\begin{abstract}
In this paper, an analytical solution is proposed for a pressurized cylindrical tube made of an orthotropic membrane, with the orthotropy directions oriented at an arbitrary angle, not necessarily parallel to the tube axis. The formulation is made in the framework of finite deformations and results in a system of three non linear equations, giving the final radius, length and rotation of the cross sections of the tube, in the deformed configuration. The set of equations is solved for balanced and unbalanced materials, with various internal pressures and orientations of the membrane. The numerical results obtained are shown to agree very well with those of an independent finite element code.
\end{abstract}

Keywords: Orthotropic membrane tube; Pressurized tube; Orthotropic elasticity

\section{Introduction}

Tensile fabrics have been widely used in textile architecture for more than half a century. Nowadays, very large structures are built as assemblies of pieces of fabrics and among the materials used, coated fabrics occupy a major place due to their interesting mechanical properties: they are light, they can be easily folded and deployed, and they are not too expensive to manufacture.

These fabrics are typically made of woven yarns encased in a PVC coating and therefore present an anisotropic behavior due to the warp and weft threads. Since they have very low bending and compressive stiffness, they can only be used in tension. In or der to use them under other loading states, it is necessary to induce a pre stress by means of air pressure, as in the so called air sup ported and air inflated structures. The subsequent discussion will be limited to air inflated structures only, also known as inflatable or pneumatic structures, which are the scope of the present paper.

The inflatable tube is one of the most simple elements in inflat able structures technology. Such a tube can be used either as a sin gle beam or assembled with others in inflatable frames and more complex structures.

The study of inflatable beams necessitates to distinguish two successive stages: (i) in the first one, the beam is subjected to a suf ficiently high internal pressure, so as to induce the pre stress in the membrane and provide the beam with a bearing capacity, (ii) in the second stage, the beam can be subjected to other external load ings which can be a combination of tensile, compressive, bending or twisting loads.

Almost all the existing studies on inflatable tubes dealt with bending or torsion loadings. Pioneering theoretical works are due to Comer and Levy [1], Douglas [2] and Webber [3], who investi gated isotropic beams using Euler Bernoulli's kinematics. Main et al. $[4,5]$ undertook experimental works on the bending of inflat able beams and took account of the biaxial state in their analysis. The shear effect in inflatable beams was considered by Steeves [6] who developed a theory based on the minimum potential en ergy principle and gave the solution in terms of Green functions, and by Fichter [7] who used Timoshenko's kinematics and obtained load deflection formulae where the pressure appears explicitly in the shear stiffness term. Adopting the same kinematics, Wielgosz and Thomas $[8,9]$ derived analytical solutions for inflated panels and tubes, by writing the equilibrium equations in the pre stressed state, and taking into account the pressure as a following force. Le van and Wielgosz [10] improved Fichter's theory by using the total Lagrangian form of the virtual work principle with finite displace ments and rotations. After linearizing the resulting nonlinear equa tions, they obtained linear formulae for isotropic beams where the pressure appears explicitly both in the bending and shear stiffness es. Recently, Apedo et al. [11] went further using a 3D Timo shenko's kinematics and derived their solutions for an orthotropic fabric in finite displacements and small rotations. This work was next extended to the buckling of an orthotropic beam by Nguyen et al. [12]. Refinements of the previous formulations en abled Nguyen et al. [13] to derive the governing nonlinear equa tions for inflatable orthotropic beams and simple formulae for the deflection and rotation. 
In all the above mentioned papers, the geometry of the inflated beam is either assumed to be known or computed by means of lin ear elasticity formulae, as in Le van and Wielgosz's paper [10] for isotropic materials, and in Apedo et al's paper [11] for orthotropic materials. Whereas such linear formulae are sufficient in the con text of small deformations, they should be replaced by more accu rate formulae when there are finite deformations, as is the case nowadays with modern fabrics which are able to bear very high tensions.

The present work is devoted to the above mentioned first stage where the beam is subjected to an internal pressure only, with the purpose of obtaining an analytical solution for a pressurized mem brane tube made of an orthotropic material. The main features of the paper are: (i) the warp or weft direction of the membrane can be oriented at an arbitrary angle, not parallel to the tube axis (for convenience, use is made of the terms 'warp' and 'weft' to des ignate the in plane orthotropy directions of the membrane); and (ii) the obtained equations hold in finite deformations, in particular finite rotations of the tube.

The paper is organized as follows. In Section 2, the problem will be formulated in finite deformations for a finite thickness ortho tropic tube and the equations will be derived considering a very small thickness with respect to the tube radius so that the tube be comes a membrane tube. This will result in a small system of three nonlinear equations, which can easily be solved by an iterative Newton type scheme to get the final geometry as well as the stres ses in the pressurized tube. In Section 3, the influence of the inter nal pressure and the orthotropy directions will be studied for two materials, one is balanced and the other unbalanced. It will be shown that when the warp or weft direction of the membrane is not parallel to the tube axis, the tube undergoes a rotation around its axis, rotation which varies as a nonlinear function of the inter nal pressure. Eventually, in Section 4, comparison with finite ele ment results will be made in order to validate the proposed theory.

Note that we consider an orthotropic homogeneous membrane, rather than a fabric which is inhomogeneous due to its complex microstructure. Phenomena specific to fabrics such as kinematics of crimp interchange, shear jamming and yarn yarn friction, will not be considered as they are out of the scope of the paper. How ever, one may expect that the results obtained can be used for fab rics to a certain extent.

\section{Analytical solution for an inflated orthotropic membrane tube}

\subsection{Definition of the problem}

The problem is formulated in finite deformations and one has to distinguish between the reference and the final configurations of the tube. Let the current position $\mathbf{x}$ of a particle of the tube in the actual state be defined by the cylindrical coordinates $r, \theta, z$, so that $\mathbf{x}=r \mathbf{e}_{r}(\theta)+z \mathbf{e}_{z}$. The current cylindrical basis at point $\mathbf{x}$ is de noted $\mathbf{b} \equiv\left(\mathbf{e}_{r}(\theta), \mathbf{e}_{\theta}(\theta), \mathbf{e}_{z}\right)$, with $\mathbf{e}_{r}$ and $\mathbf{e}_{\theta}$ parallel to the radial and circumferential directions, respectively.
The position $\mathbf{X}$ of the same particle in the reference state is de fined by the initial values of $r, \theta$ and $z$, denoted by $R, \Theta$ and $Z$, so that $\mathbf{X}=\operatorname{Re}_{r}(\Theta)+Z \mathbf{e}_{z}$. The reference cylindrical basis at point $\mathbf{X}$ is $\mathbf{B} \equiv\left(\mathbf{e}_{r}(\Theta), \mathbf{e}_{\theta}(\Theta), \mathbf{e}_{z}\right)$

The reference geometry is a thick walled tube of axis $O \mathbf{e}_{\mathrm{z}}$, inner radius $A$, outer radius $B$, thickness $H=B \quad A$, length $L$, and closed at the ends $Z=0$ and $Z=L$, as shown in Fig. 1 .

It is assumed that the tube is made of an orthotropic material with the orthotropy basis $\left(\mathbf{e}_{n}, \mathbf{e}_{\ell}, \mathbf{e}_{t}\right)$ in the reference configuration. The normal direction $\mathbf{e}_{n}$ is equal to $\mathbf{e}_{r}(\Theta)$ and the angle (around $\mathbf{e}_{r}(\Theta)$ ) between the longitudinal direction $\mathbf{e}_{\ell}$ and the tube axis $\mathbf{e}_{z}$ is denoted $\alpha, 0 \leqslant \alpha \leqslant 180^{\circ}$.

The tube is free of stress in the reference configuration and let it be subjected to an internal pressure $p$.

\subsection{Deformation}

It is assumed that the cross sections of the pressurized tube re main planar and perpendicular to axis $O \mathbf{e}_{z}$, in such a way that the tube remains cylindrical in the deformed state. The deformed geometry is defined by the inner radius $a$, outer radius $b$, thickness $h=b \quad a$ and length $\ell$, which correspond to their initial values A, B, $\mathrm{H}$ and $\mathrm{L}$, respectively. The deformation of the tube is then defined by the following relation giving the final cylindrical coordinates $r$, $\theta, z$ as functions of the initial coordinates $R, \Theta, Z$ :

$\begin{array}{llllll}r & r(R) & \theta & \Theta+\beta(R, Z) & Z & Z(Z)\end{array}$

Functions $r(R)$ and $z(Z)$ correspond to the radial and axial displacements, whereas function $\beta(R, Z)$ has been introduced in order to represent the circumferential displacement or the rota tion of the cross sections around the tube axis, due to the fact that the orthotropy directions do not coincide with the cylindrical axes. The functions $\beta(R, Z)$ and $z(Z)$ have to satisfy $\beta(R, 0)=0$ and $z(0)=0$ if one considers that the end section $Z=0$ does not rotate.

\subsection{Deformation gradient Strains}

Since the current position $\mathbf{x}$ of a particle of the tube is resolved in terms of the reference basis $\mathbf{B}$ via $\mathbf{x}=r \cos \beta$ $\mathbf{e}_{r}(\Theta)+r \sin \beta \mathbf{e}_{\theta}(\Theta)+z \mathbf{e}_{z}$, the matrix of the deformation gradient tensor can be expressed in basis $\mathbf{B}$ as

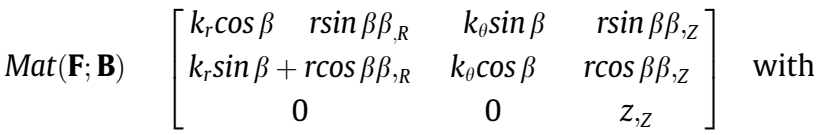

$k_{r} \equiv \frac{d r}{d R}, \quad k_{\theta} \equiv \frac{r}{R}>0$

The bijectivity condition $J=\operatorname{det} \mathbf{F}=k_{r} k_{\theta} z, Z>0$ leads to

$k_{r} z_{, z}>0$

The matrix of the Green strain tensor $\mathbf{E}=\left(\begin{array}{ll}\mathbf{F}^{T} \mathbf{F} & \mathbf{I}\end{array}\right) / 2$ in the reference basis is derived from Relation (2):
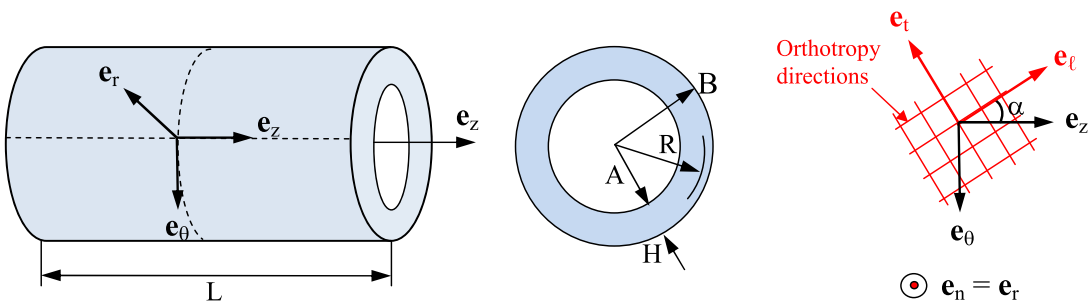

$\odot \mathbf{e}_{\mathrm{n}}=\mathbf{e}_{\mathrm{r}}$

Fig. 1. Reference geometry of the tube. 


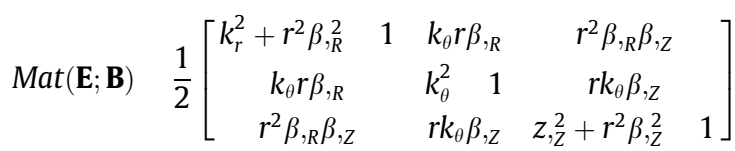

The components of the Green strain tensor $\mathbf{E}$ in the reference basis do not depend on the cylindrical coordinate $\Theta$.

\subsection{Stresses}

When the tube is very thin, it behaves as a membrane tube made of an orthotropic material, with the orthotropy directions $\mathbf{e}_{\ell}$ and $\mathbf{e}_{t}$ parallel to the warp and weft directions, and the orienta tion of the membrane is defined by the angle $\alpha$ between $\mathbf{e}_{\ell}$ and $\mathbf{e}_{z}$. The relationship between the stresses and the strains in the mem brane can be derived from a micro mechanical approach, see, e.g., [14] and [15], dealing with the local interaction between the yarns, the interaction between the yarns and the coating, as well as the friction between the warp and weft yarns. However, since such an approach involves a great number of parameters it can hardly be used in structural mechanics, and an alternative way usually adopted in the literature is to use a phenomenological constitutive law. Many works on inflatable beams were conducted in small deformations using a linear elastic constitutive law. In another context, Galliot and Luchsinger [16] undertook experiments on PVC coated polyester fabrics using a biaxial machine, for 13 load ratios varying from $11: 1$ to $1: 11$. By adopting a linear elastic ortho tropic constitutive law, they determined the elastic constants for each given load ratio. In the case of the inflated tube in hand, the ratio between the longitudinal and transversal loads is $1: 2$.

The approach chosen in the present work consists in stating the problem for a finite thickness tube and then deriving the govern ing equations for a thin tube $(H \ll A)$. It is assumed that the 3D constitutive law for the thick tube is the hyperelastic Saint Venant Kirchhoff law E $\mathbb{C}: \boldsymbol{\Sigma}$, where the symbol ':' denotes the double contraction between the compliance tensor $\mathbb{C}$ (fourth order tensor) and the second Piola Kirchhoff stress tensor $\boldsymbol{\Sigma}$ (second order ten sor). In a consistent way with what is made on the thin tube, the compliance tensor $\mathbb{C}$ is that of an orthotropic material and the con stitutive law is written in the orthotropy basis $\left(\mathbf{e}_{n}, \mathbf{e}_{\ell}, \mathbf{e}_{t}\right)$ as follows:

$\left\{\begin{array}{c}E_{n n} \\ E_{\ell \ell} \\ E_{t t} \\ 2 E_{\ell t} \\ 2 E_{t n} \\ 2 E_{n \ell}\end{array}\right\}=\left[\begin{array}{ccc|ccc}C_{n n n n} & C_{n n \ell \ell} & C_{n n t t} & 0 & 0 & 0 \\ C_{\ell \ell n n} & C_{\ell \ell \ell \ell} & C_{\ell \ell t t} & 0 & 0 & 0 \\ C_{t t n n} & C_{t t \ell \ell} & C_{t t t t} & 0 & 0 & 0 \\ \hline 0 & 0 & 0 & C_{\ell t t \ell} & 0 & 0 \\ 0 & 0 & 0 & 0 & C_{t n n t} & 0 \\ 0 & 0 & 0 & 0 & 0 & C_{n \ell \ell n}\end{array}\right]\left\{\begin{array}{c}\Sigma_{n n} \\ \Sigma_{\ell \ell} \\ \Sigma_{t t} \\ \Sigma_{\ell t} \\ \Sigma_{t n} \\ \Sigma_{n \ell}\end{array}\right\}$

where

$$
\begin{array}{lccccccc}
C_{n n n n} & \frac{1}{E_{n}} & C_{n n \ell \ell} & C_{\ell \ell n n} & \frac{v_{\ell n}}{E_{\ell}} & C_{n n t t} & C_{t t n n} & \frac{v_{t n}}{E_{t}} \\
C_{\ell \ell \ell \ell} & \frac{1}{E_{\ell}} & C_{\ell \ell t t} & C_{t t \ell \ell} & \frac{v_{\ell t}}{E_{\ell}} & C_{t t t t} & \frac{1}{E_{t}} & \\
C_{\ell t t \ell} & \frac{1}{G_{\ell t}} & C_{t n n t} & \frac{1}{G_{t n}} & C_{n \ell \ell n} & \frac{1}{G_{n \ell}} &
\end{array}
$$

The constitutive law in the reference basis $\mathbf{B}$ necessitates a change of basis since the orthotropy basis is different to basis $\mathbf{B}$. The compliance matrix $\operatorname{Mat}(\mathbb{C} ; \mathbf{B})$ in the reference basis can be de rived from the compliance matrix $\operatorname{Mat}\left(\mathbb{C} ; \mathbf{e}_{n}, \mathbf{e}_{\ell}, \mathbf{e}_{t}\right)$ in the orthotro py basis, Eq. (5), by

$\forall i, j, k, \ell \in\{r, \theta, z\}, C_{i j k \ell} \quad P_{i I} P_{j J} P_{k K} P_{\ell L} C_{I J K L}$

where there is sums on the repeated indices $I, J, K, L \in\{n, \ell, t\}$ and $[P]$ is the change of basis matrix from reference basis $\mathbf{B}$ to the orthotro py basis $\left(\mathbf{e}_{n}, \mathbf{e}_{\ell}, \mathbf{e}_{t}\right)$ :
$[P]=\begin{gathered}e_{n} \\ e_{r}(\Theta) \\ e_{\theta}(\Theta) \\ e_{z}\end{gathered}\left[\begin{array}{ccc}1 & 0 & e_{t} \\ 0 & -\sin \alpha & -\cos \alpha \\ 0 & \cos \alpha & -\sin \alpha\end{array}\right]$

The change of basis formula (7) leads to the constitutive law in the reference basis $\mathbf{B}$ :

$\left\{\begin{array}{l}E_{r r} \\ E_{\theta \theta} \\ E_{z z} \\ 2 E_{\theta z} \\ 2 E_{z r} \\ 2 E_{r \theta}\end{array}\right\} \quad\left[\begin{array}{cccccc}C_{r r r r} & C_{r r \theta \theta} & C_{r r z z} & C_{r r z \theta} & 0 & 0 \\ C_{\theta \theta r r} & C_{\theta \theta \theta \theta} & C_{\theta \theta z z} & C_{\theta \theta z \theta} & 0 & 0 \\ C_{z z r r} & C_{z z \theta \theta} & C_{z z z z} & C_{z z z \theta} & 0 & 0 \\ C_{\theta z r r} & C_{\theta z \theta \theta} & C_{\theta z z z} & C_{\theta z z \theta} & 0 & 0 \\ 0 & 0 & 0 & 0 & C_{z r r z} & C_{z r \theta r} \\ 0 & 0 & 0 & 0 & C_{r \theta r z} & C_{r \theta \theta r}\end{array}\right]\left\{\begin{array}{c}\Sigma_{r r} \\ \Sigma_{\theta \theta} \\ \Sigma_{z z} \\ \Sigma_{\theta z} \\ \Sigma_{z r} \\ \Sigma_{r \theta}\end{array}\right\}$

where the compliance components in the reference basis $\mathbf{B}$ are gi ven by (we denote $c=\cos \alpha, s=\sin \alpha$ for brevity)

$$
\begin{aligned}
& C_{\text {rrrr }} C_{n n n n}
\end{aligned}
$$

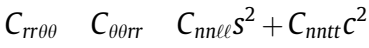

$$
\begin{aligned}
& C_{\text {rrzz }} \quad C_{z z r r} \quad C_{n n \ell \ell} C^{2}+C_{n n t t} S^{2} \\
& C_{\text {rrze }} \quad C_{\theta z r r} \quad 2\left(C_{n n \ell e} \quad C_{n n t t}\right) s C \\
& C_{\theta \theta \theta \theta} \quad C_{\ell \ell \ell \ell} s^{4}+C_{t t t t} c^{4}+\left(C_{\ell t t \ell}+2 C_{\ell \ell t t}\right) s^{2} c^{2} \\
& C_{\theta \theta z z} \quad C_{z z \theta \theta} \quad\left(C_{\ell \ell \ell \ell}+C_{t t t t} \quad C_{\ell t t \ell}\right) s^{2} c^{2}+C_{\ell \ell t t}\left(s^{4}+c^{4}\right) \\
& C_{\theta \theta z \theta} \quad C_{\theta z \theta \theta} \quad 2\left(C_{\ell \ell \ell \ell} s^{3} c \quad C_{t t t t} c^{3} s\right)+2 C_{\ell \ell t t}\left(s^{3} c \quad c^{3} s\right) \quad C_{\ell t t \ell}\left(\begin{array}{ll}
c^{3} s & s^{3} c
\end{array}\right. \\
& C_{z z z z} \quad C_{\ell \ell \ell \ell} c^{4}+C_{t t t t} s^{4}+\left(C_{\ell t t \ell}+2 C_{\ell \ell t t}\right) s^{2} c^{2} \\
& C_{z z z \theta} \quad C_{\theta z z z} \quad 2\left(C_{\ell \ell \ell \ell} c^{3} s \quad C_{t t t t} s^{3} c\right) \quad 2 C_{\ell \ell t t}\left(s^{3} c \quad c^{3} s\right)+C_{\ell t t \ell}\left(c^{3} s \quad s^{3} c\right) \\
& C_{\theta z z \theta} \quad 4\left(C_{\ell \ell \ell \ell}+C_{t t t t} \quad 2 C_{\ell \ell t t}\right) s^{2} c^{2}+C_{\ell t t \ell}\left(c^{2} \quad s^{2}\right)^{2} \\
& C_{\text {zrrz }} \quad s^{2} C_{\text {tnnt }}+c^{2} C_{\text {nenn }}
\end{aligned}
$$

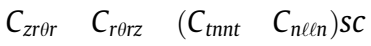

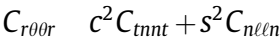

It can be seen from Relations (4) and (9) that the components of the stress tensor $\boldsymbol{\Sigma}$ in the reference basis do not depend on the cylindri cal coordinate $\Theta$.

On the other hand, the second Piola Kirchoff stress tensor $\boldsymbol{\Sigma}$ is related to the Cauchy stress tensor $\boldsymbol{\sigma}$ via

$\Sigma \quad J \mathbf{F}^{1} \boldsymbol{\sigma} \mathbf{F}^{T}$

Thus, the matrix of the second Piola Kirchhoff stress is computed by

$\operatorname{Mat}(\boldsymbol{\Sigma} ; \mathbf{B}) \quad J(\operatorname{Mat}(\mathbf{F} ; \mathbf{B}, \mathbf{b}))^{1} . \operatorname{Mat}(\boldsymbol{\sigma} ; \mathbf{b}) .(\operatorname{Mat}(\mathbf{F} ; \mathbf{b}, \mathbf{B}))^{T}$

where the Cauchy stress matrix is written in the current basis $\mathbf{b}$ as

$\operatorname{Mat}(\boldsymbol{\sigma} ; \mathbf{b}) \quad\left[\begin{array}{ccc}\sigma_{r r} & \sigma_{r \theta} & \sigma_{r z} \\ \sigma_{\theta r} & \sigma_{\theta \theta} & \sigma_{\theta z} \\ \sigma_{z r} & \sigma_{z \theta} & \sigma_{z z}\end{array}\right]$

and matrix $\operatorname{Mat}(\mathbf{F} ; \mathbf{B}, \mathbf{b})$ is obtained from matrix $\operatorname{Mat}(\mathbf{F} ; \mathbf{B})$ in Rela tion (2) by

$\operatorname{Mat}(\mathbf{F} ; \mathbf{B}, \mathbf{b}) \quad \operatorname{Mat}(\mathbf{F} ; \mathbf{B}) .\left[\begin{array}{ccc}\cos \beta & \sin \beta & 0 \\ \sin \beta & \cos \beta & 0 \\ 0 & 0 & 1\end{array}\right]$

One derives the components of the second Piola Kirchhoff stress in basis $\mathbf{B}$ in terms of the components of the Cauchy stress and the kinematic unknowns $r(R), \beta(R, Z), z(Z)$ : 


$$
\begin{aligned}
& \Sigma_{r r} \frac{k_{\theta} z_{, Z}}{k_{r}} \sigma_{r r} \\
& \Sigma_{r \theta} \quad z_{, Z} \sigma_{r \theta} \quad r \beta,{ }_{, Z} \sigma_{r z} \quad \frac{r \beta,{ }_{R} z, z}{k_{r}} \sigma_{r r} \\
& \Sigma_{r z} \quad k_{\theta} \sigma_{r z} \\
& \Sigma_{\theta \theta} \quad \frac{k_{r} Z,_{Z}}{k_{\theta}} \sigma_{\theta \theta} \quad 2 R k_{r} \beta,{ }_{Z} \sigma_{\theta z}+\frac{r \beta,{ }_{Z}^{2} R k_{r}}{z_{, z}} \sigma_{z z}+\frac{z_{, Z} r R \beta,_{R}^{2}}{k_{r}} \sigma_{r r} \\
& 2 R z,{ }_{, z} \beta,{ }_{R} \sigma_{r \theta}+2 r R \beta,_{R} \beta,{ }_{z} \sigma_{r z} \\
& \Sigma_{\theta z} \quad k_{r} \sigma_{\theta z} \quad \frac{r \beta,{ }_{2} k_{r}}{z, z} \sigma_{z z} \quad r \beta,_{R} \sigma_{r z} \\
& \Sigma_{z z} \frac{k_{r} k_{\theta}}{z_{, z}} \sigma_{z z}
\end{aligned}
$$

\subsection{Equilibrium equations}

Relations (11) or (12) show that the components of the Cauchy stress tensor $\boldsymbol{\sigma}$ do not depend on the cylindrical coordinate $\theta$. Conse quently, the equilibrium equations $\operatorname{div} \boldsymbol{\sigma}=\mathbf{0}$ write in the current ba sis b

$$
\begin{aligned}
& \sigma_{r r, r}+\frac{1}{r}\left(\sigma_{r r} \quad \sigma_{\theta \theta}\right)+\sigma_{r z, z} \quad 0 \\
& \sigma_{\theta r, r}+\frac{2}{r} \sigma_{r \theta}+\sigma_{\theta z, z} \quad 0 \\
& \sigma_{z r, r}+\frac{1}{r} \sigma_{z r}+\sigma_{z z, z} \quad 0
\end{aligned}
$$

\subsection{Assumptions for a thin tube}

Let us now assume that the initial tube thickness $H$ is very small compared with the initial radius $A$, which enables one to make the following assumptions:

Assumption 1. $k_{r}$ and $k_{\theta}$ do not depend on $R$, i.e. they are constant through the tube thickness. Indeed, since the tube is very thin, radius $R$ lies within the very small interval $[A, A+H]$, and functions $k_{r}(R)$ and $k_{\theta}(R)$ defined in (2) can be assumed constant over this interval.

Assumption 2. The rotation angle $\beta(R, Z)$ does not depend on $R$ either. Furthermore, it is assumed to depend linearly on $Z: \beta(Z)=$ $k_{\beta} Z$, where $k_{\beta}$ is a constant scalar.

Assumption 3. The final coordinate $z$ is assumed to depend line arly on $Z$ : $z(Z)=k_{z} Z$, where $k_{z}>0$ is another constant scalar.

Under these assumptions, the Green strain matrix given in Rela tion (4) simplifies as follows

$\operatorname{Mat}(\mathbf{E} ; \mathbf{B}) \quad \frac{1}{2}\left[\begin{array}{ccccc}k_{r}^{2} & 1 & 0 & 0 & \\ 0 & k_{\theta}^{2} & 1 & r k_{\beta} k_{\theta} & \\ 0 & r k_{\beta} k_{\theta} & k_{z}^{2}+r^{2} k_{\beta}^{2} & 1\end{array}\right]$

Relation (14) shows that the components of the Green strain tensor $\mathbf{E}$ in the reference basis do not depend on $Z$. By virtue of Relations (9) and (12), the components of the second Piola Kirchhoff stress tensor $\boldsymbol{\Sigma}$ are independent of $Z$, whereas those of Cauchy stress ten sor $\boldsymbol{\sigma}$ are independent of $z$.

Consider the relationship

$\frac{|d \mathbf{X}|^{2}}{|d \mathbf{X}|^{2}} \quad \mathbf{N} \cdot \mathbf{C N}$

where $\mathbf{C}=\mathbf{2} \mathbf{E}+\mathbf{I}$ is the right Cauchy deformation tensor, $d \mathbf{X}$ is a material line element and $d \mathbf{x}$ its spatial counterpart. If one takes
$\mathbf{N}=\mathbf{e}_{r}(\Theta)$, then Relation (15) gives $\frac{|d \mathbf{x}|^{2}}{|d \mathbf{X}|^{2}} \quad \mathbf{e}_{r}(\Theta) \cdot \mathbf{C}_{r}(\Theta) \quad k_{r}^{2}$, which is constant in view of Assumption 1 above. Consequently, Relation (15) holds for any vectors $d \mathbf{X}$ and $d \mathbf{x}$ parallel to $\mathbf{e}_{r}(\Theta)$, not necessar ily of infinitesimal lengths. Thus, it can be applied to a point on the internal wall of the membrane $R=A$, with $d \mathbf{X}=H \mathbf{e}_{r}(\Theta), \mathbf{N}=\mathbf{e}_{r}(\Theta)$ and $d \mathbf{x}=h \mathbf{e}_{r}(\theta)$, to give

$\frac{h^{2}}{H^{2}} \quad \mathbf{e}_{r}(\Theta) \cdot \mathbf{C e}_{r}(\Theta) \quad k_{r}^{2}$

From $k_{z}>0$ and the bijectivity condition (3), one gets $k_{r}>0$ and thus $\frac{h}{H} \quad k_{r}$. The final thickness radius ratio $h / a$ is related to the initial ra tio $H / A$ by

$\frac{h}{a} \quad \frac{h}{H} \frac{H}{A} \frac{A}{a} \quad \frac{H}{A} \frac{k_{r}}{k_{\theta}}$

In the case of an inflated tube, $k_{\theta} \quad \frac{a}{A} \geqslant 1$ and $k_{r} \quad \frac{h}{H}$ is bounded, hence the ratio $\frac{k_{r}}{k_{\theta}}$ is bounded too. Relation (16) shows that $H \ll A$ leads to $h \ll a$ : the final thickness $h$ is very small compared with the final radius $a$. This justifies the following assumption:

Assumption 4. All the Cauchy stress components except $\sigma_{r r}$ are constant through the tube thickness. The axial stress $\sigma_{r r}$, which must satisfy $\sigma_{r r}(a)=p$ and $\sigma_{r r}(b)=0$, is assumed to be bounded by $p \leqslant \sigma_{r r} \leqslant 0$ and negligible with respect to the hoop and the axial stresses: $\left|\sigma_{r r}\right| \ll \sigma_{\theta \theta}$ and $\left|\sigma_{r r}\right| \ll \sigma_{z z}$.

\subsection{Set of equations for an inflated orthotropic thin tube}

Applying Assumption 4 to the fact that the stresses $\sigma_{\theta r}, \sigma_{z r}$ are zero on the inner and outer walls, one obtains $\sigma_{\theta r}=\sigma_{z r}=0$ through out. By writing the moment balance with respect to the tube axis $O \mathbf{e}_{z}$, one easily finds $\sigma_{\theta z}=0$ since there is no external twisting tor que on the tube. Hence, the Cauchy stress matrix is of the following form

\begin{tabular}{|c|c|c|c|}
\hline Initial geometry & Initial length $L$ & $1 \mathrm{~m}$ & \multirow[b]{3}{*}{$\begin{array}{l}\text { Balanced } \\
\text { material }\end{array}$} \\
\hline \multirow[b]{2}{*}{ Material properties } & $\begin{array}{l}\text { Initial radius } A \\
\text { Initial thickness } H\end{array}$ & $\begin{array}{l}0.06 \mathrm{~m} \\
0.6 \mathrm{~mm}\end{array}$ & \\
\hline & & $\begin{array}{l}\text { Unbalanced } \\
\text { material }\end{array}$ & \\
\hline & $\begin{array}{l}\text { Warp Young's } \\
\text { modulus } E_{\ell} H(\mathrm{kN} / \mathrm{m})\end{array}$ & 780 & 660 \\
\hline & $\begin{array}{l}\text { Weft Young's } \\
\text { Modulus } E_{t} H(\mathrm{kN} / \mathrm{m})\end{array}$ & 504 & 660 \\
\hline & $\begin{array}{l}\text { In-plane shear } \\
\text { modulus } G_{\ell t} H(\mathrm{kN} / \mathrm{m})\end{array}$ & 13.8 & 9 \\
\hline & Poisson ratio $v_{\ell t}$ & 0.31 & 0.23 \\
\hline & Poisson ratio $v_{t \ell}$ & 0.2 & 0.23 \\
\hline $\begin{array}{l}\text { Orientation angle } \alpha \text { of } \\
\text { the membrane }\end{array}$ & & $0-180^{\circ}$ & $0-90^{\circ}$ \\
\hline \multirow{2}{*}{$\begin{array}{l}\text { Internal pressure } p \\
\quad(\mathrm{kPa})\end{array}$} & 5075100125150 & & \\
\hline & 175200 & & \\
\hline
\end{tabular}

$\operatorname{Mat}(\boldsymbol{\sigma} ; \mathbf{b}) \quad\left[\begin{array}{ccc}0 & 0 & 0 \\ 0 & \sigma_{\theta \theta} & 0 \\ 0 & 0 & \sigma_{z z}\end{array}\right]$

which does satisfy the equilibriums $(13)_{b}(13)_{c}$. The boundary con dition $\int_{S(z,)} \sigma_{z z} d S p \pi a^{2}$ on the end cross section $z=\ell$ yields $\sigma_{z z} \pi\left(b^{2} a^{2}\right)=p \pi a^{2}$ and then

$\sigma_{z z} \quad \frac{p a}{2 h} \quad \frac{p A}{2 H} \frac{k_{\theta}}{k_{r}}$

Table 1

Numerical values for the orthotropic tube. 
Integrating the equilibrium Eq. (13) from $r=a$ to $r=b$ and taking account of the boundary condition $\sigma_{r r}(r=a)=p$ and $\sigma_{r r}(r=b)=0$ on the inner and outer walls give

$\sigma_{\theta \theta} \quad \frac{p a}{h} \quad \frac{p A}{H} \frac{k_{\theta}}{k_{r}}$

Inserting Relation (17) into (12) leads to the second Piola Kirchhoff stress

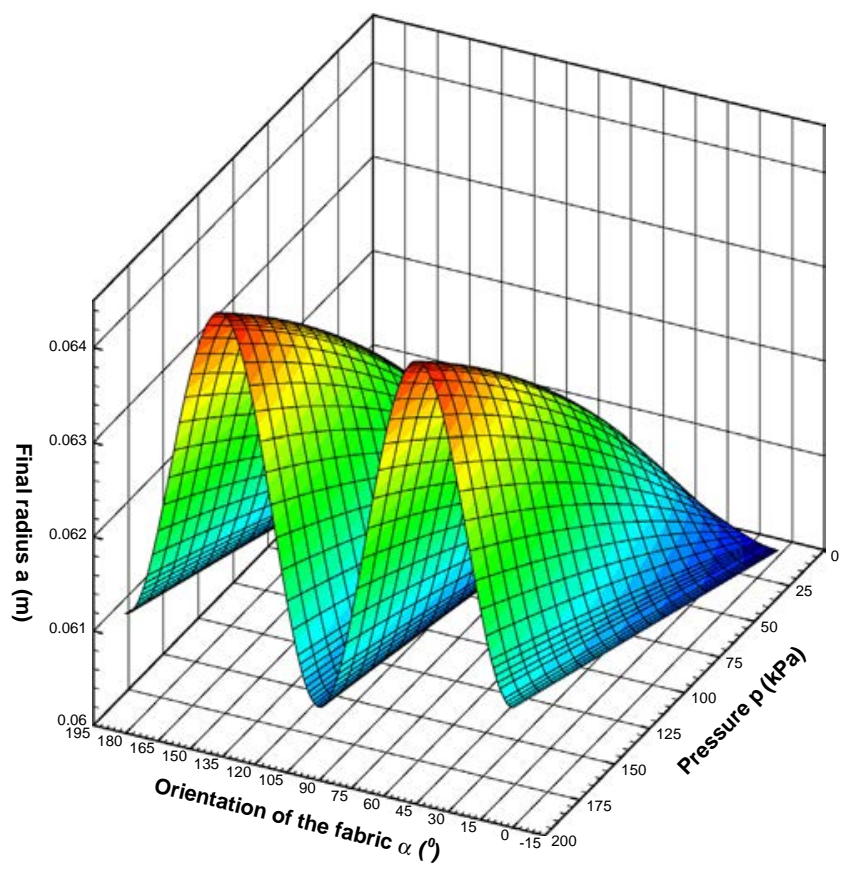

(a) Final radius $a$
$\operatorname{Mat}(\boldsymbol{\Sigma} ; \mathbf{B}) \quad\left[\begin{array}{ccc}0 & 0 & 0 \\ 0 & \frac{r^{2} k_{r} k_{\beta}^{2}}{k_{\theta} k_{z}} \sigma_{z z}+\frac{k_{r} k_{z}}{k_{\theta}} \sigma_{\theta \theta} & \frac{r k_{r} k_{\beta}}{k_{z}} \sigma_{z z} \\ 0 & \frac{r k_{r} k_{\beta}}{k_{z}} \sigma_{z z} & \frac{k_{r} k_{\theta}}{k_{z}} \sigma_{z z}\end{array}\right]$

which shows that the second Piola Kirchhoff stress is in plane stress state with respect to plane $\left(\mathbf{e}_{\theta}(\Theta), \mathbf{e}_{z}\right)$. According to (20), the constitutive law (9) reduces to the following form for the prob lem of the tube in hand

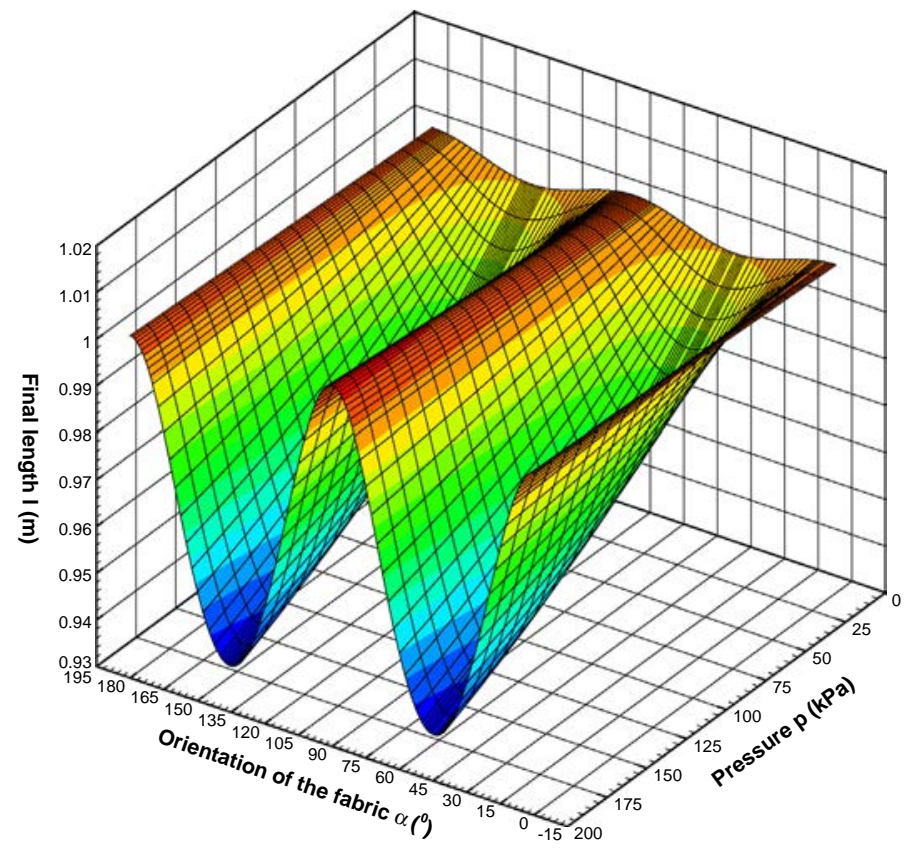

(b) Final length $\ell$

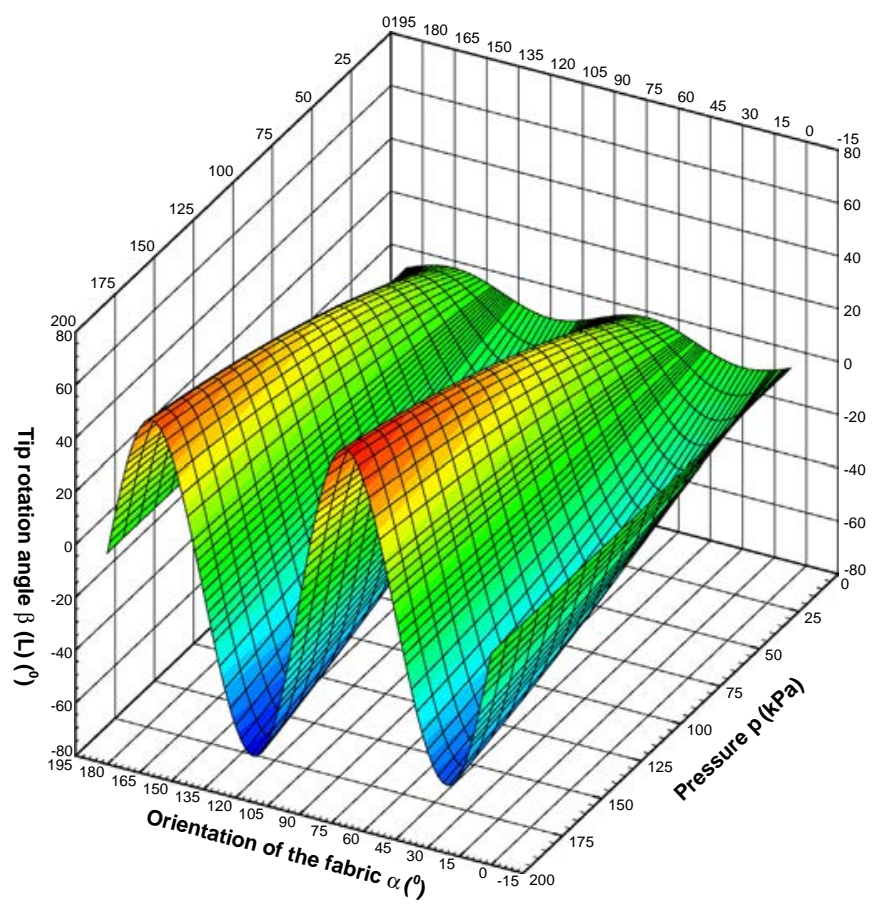

(c) Tip rotation angle $\beta(L)$

Fig. 2. Change of geometry and tip rotation of the tube - Unbalanced material. 
$\left\{\begin{array}{l}E_{\theta \theta} \\ E_{z z} \\ 2 E_{\theta z}\end{array}\right\} \quad\left[\begin{array}{lll}C_{\theta \theta \theta \theta} & C_{\theta \theta z z} & C_{\theta \theta z \theta} \\ C_{z z \theta \theta} & C_{z z z z} & C_{z z z \theta} \\ C_{\theta z \theta \theta} & C_{\theta z z z} & C_{\theta z z \theta}\end{array}\right]\left\{\begin{array}{c}\Sigma_{\theta \theta} \\ \Sigma_{z z} \\ \Sigma_{\theta z}\end{array}\right\}$

Eventually, by replacing the left hand side of (21) by (14) and the right hand side by (18) (20) with $r=k_{\theta} A$, one obtains three equa tions for three unknowns $k_{z}, k_{\theta}$ and $k_{\beta}$ :

$$
\begin{aligned}
& \left(\begin{array}{ll}
k_{\theta}^{2} & 1
\end{array}\right) k_{z} \quad p A\left[C_{\theta \theta \theta \theta}^{*}\left(A^{2} k_{\theta}^{2} k_{\beta}^{2}+2 k_{z}^{2}\right)+C_{\theta \theta z z}^{*} k_{\theta}^{2} \quad C_{\theta \theta z \theta}^{*} A k_{\theta}^{2} k_{\beta}\right] \\
& {\left[k_{z}^{2}+\left(A k_{\theta} k_{\beta}\right)^{2} \quad 1\right] k_{z} \quad p A\left[C_{z z \theta \theta}^{*}\left(A^{2} k_{\theta}^{2} k_{\beta}^{2}+2 k_{z}^{2}\right)+C_{z z z z}^{*} k_{\theta}^{2} \quad C_{z z z \theta}^{*} A k_{\theta}^{2} k_{\beta}\right.} \\
& 2 A k_{\theta}^{2} k_{\beta} k_{z} \quad p A\left[C_{\theta z \theta \theta}^{*}\left(A^{2} k_{\theta}^{2} k_{\beta}^{2}+2 k_{z}^{2}\right)+C_{\theta z z z}^{*} k_{\theta}^{2} \quad C_{\theta z z \theta}^{*} A k_{\theta}^{2} k_{\beta}\right]
\end{aligned}
$$

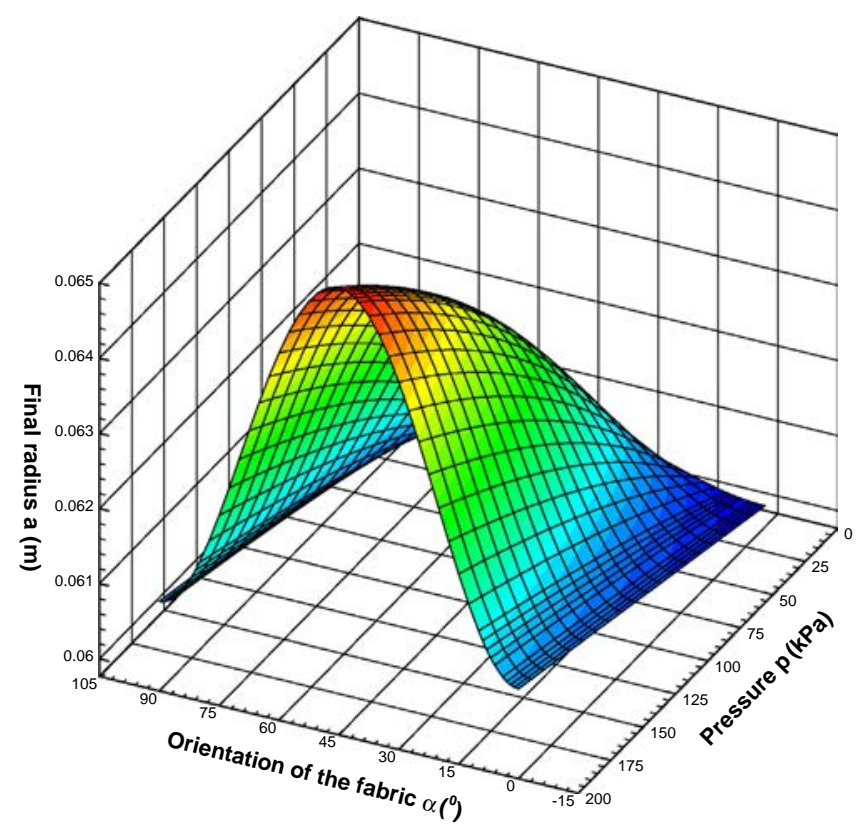

(a) Final radius $a$ where $\mathbb{C}^{*} \equiv \mathbb{C} / H$. Given the initial radius $A$, the compliance coeffi cients in the orthotropy basis (6) of the membrane, the orientation angle $\alpha$ of the membrane and the internal pressure $p$, the nonlinear system (22) can easily be solved by means of Newton Raphson's iterative scheme.

Once $k_{z}, k_{\theta}$ and $k_{\beta}$ are obtained, the final geometry of the inflated tube is known final length $\ell=k_{z} L$, final radius $a=k_{\theta} A$ as well as the rotation of the tube $\beta(Z)=k_{\beta} Z$. The integrated Cauchy stresses on the tube thickness are computed from (18) and (19): $h \sigma_{\theta \theta}=2$ $h \sigma_{z z}=p A k_{\theta}$.

In the case when the orthotropy directions of the membrane coincide with the circumference and the axis of the tube, i.e. $\alpha=0^{\circ}, \quad 90^{\circ}$ or $180^{\circ}$, one has $\sin \alpha \cos \alpha=0$ and $C_{\theta \theta z \theta}^{*} \quad C_{\theta z \theta \theta}^{*} \quad C_{\theta z z z}^{*} \quad C_{z z z \theta}^{*} \quad 0$ from Relation (10). Consequently, the system (22) simplifies to

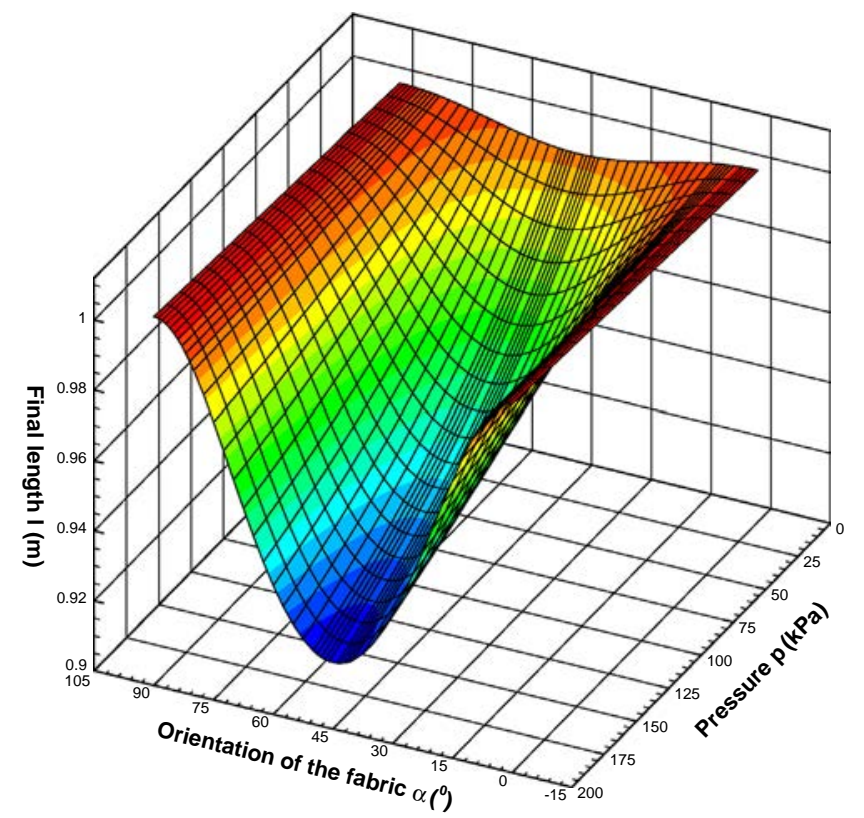

(b) Final length $\ell$

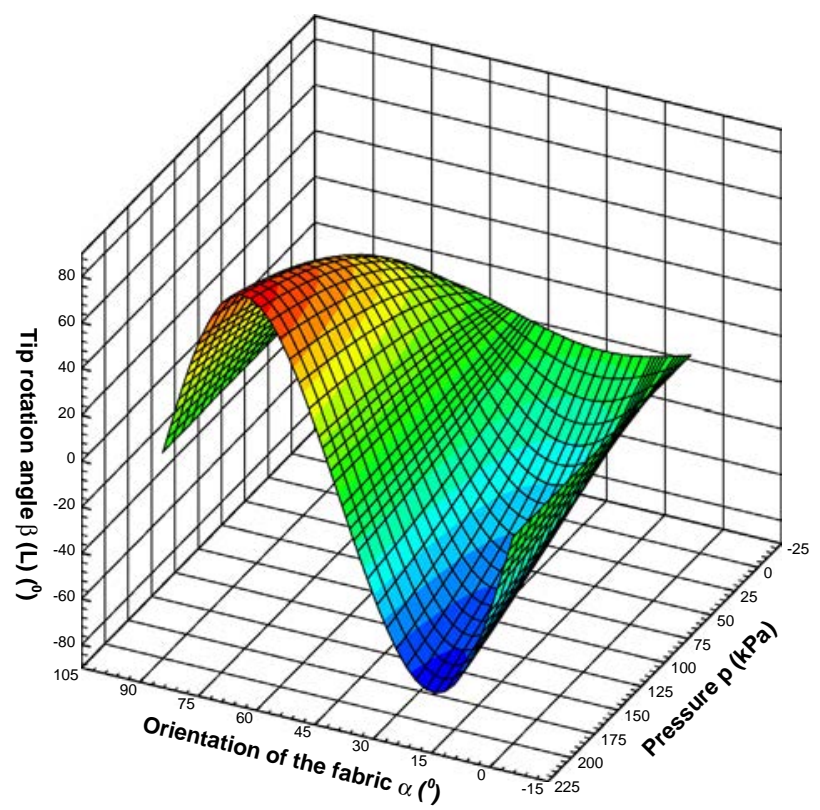

(c) Tip rotation angle $\beta(L)$

Fig. 3. Change of geometry and tip rotation of the tube - Balanced material. 
$\left(\begin{array}{ll}k_{\theta}^{2} & 1\end{array}\right) k_{z} \quad p A\left[C_{\theta \theta \theta \theta}^{*}\left(A^{2} k_{\theta}^{2} k_{\beta}^{2}+2 k_{z}^{2}\right)+C_{\theta \theta z z}^{*} k_{\theta}^{2}\right]$

$\left[\begin{array}{ll}k_{z}^{2}+\left(A k_{\theta} k_{\beta}\right)^{2} & 1\end{array}\right] k_{z} \quad p A\left[C_{z z \theta \theta}^{*}\left(A^{2} k_{\theta}^{2} k_{\beta}^{2}+2 k_{z}^{2}\right)+C_{z z z z}^{*} k_{\theta}^{2}\right]$

$2 A k_{\theta}^{2} k_{\beta} k_{z} \quad p A^{2} k_{\theta}^{2} k_{\beta} C_{\theta z z \theta}^{*}$

From (23) , one gets $k_{\beta} A k_{\theta}^{2}\left(2 k_{z}+p A C_{\theta z z \theta}^{*}\right) \quad 0$ and thus, since $A k_{\theta}^{2}\left(2 k_{z}+p A C_{\theta z z \theta}^{*}\right)>0$, one finds that $k_{\beta}=0$, which means that the tube does not rotate as one might expect. Inserting $k_{\beta}=0$ into $(23)_{a} b_{b}$ then yields two equations for two unknowns $k_{\theta}$ and $k_{z}$

$$
\begin{aligned}
& \left(\begin{array}{ll}
k_{\theta}^{2} & 1
\end{array}\right) k_{z} \quad p A\left(2 C_{\theta \theta \theta \theta}^{*} k_{z}^{2}+C_{\theta \theta z z}^{*} k_{\theta}^{2}\right) \\
& \left(\begin{array}{ll}
k_{z}^{2} & 1
\end{array}\right) k_{z} \quad p A\left(2 C_{z z \theta \theta}^{*} k_{z}^{2}+C_{z z z z}^{*} k_{\theta}^{2}\right)
\end{aligned}
$$

One may eliminate $k_{\theta}$ between the above equations in order to ob tain one cubic equation with unknown $k_{z}$. This particular case has been treated in a previous work [17].

\section{Numerical results}

Here, the nonlinear system (22) is solved for two types of mate rials: (1) the unbalanced material with different Young's moduli in the warp and weft directions; and (2) the balanced material with the same Young's modulus in the warp and weft directions. The numerical values of the material coefficients should be chosen to be somewhat realistic, for this purpose we have chosen values sim ilar to those of coated fabrics F702 and V700 which were identified experimentally by Galliot and Luchsinger on a biaxial machine. The material properties are displayed in Table 1 . The orientation angle $\alpha$ of the membrane is taken between $0^{\circ}$ and $180^{\circ}$ for the unbal anced material and between $0^{\circ}$ and $90^{\circ}$ for the balanced material. The internal pressure $p$ ranges from 50 to $200 \mathrm{kPa}$ by steps of $25 \mathrm{kPa}$.

Figs. 2 and 3 depict the final radius and length together with the tip rotation of the inflated tube versus the orientation angle $\alpha$ and the internal pressure $p$ for the two materials, respectively.

\subsection{Change of geometry and rotation versus the orientation of the membrane}

In this section, we examine the final geometry (radius $a$, length $\ell$ ) and the tip rotation angle $\beta(L)$ of the inflated tube as functions of the orientation of the membrane. The internal pressure $p$ is given a fixed value, $p=50,100$ or $200 \mathrm{kPa}$.

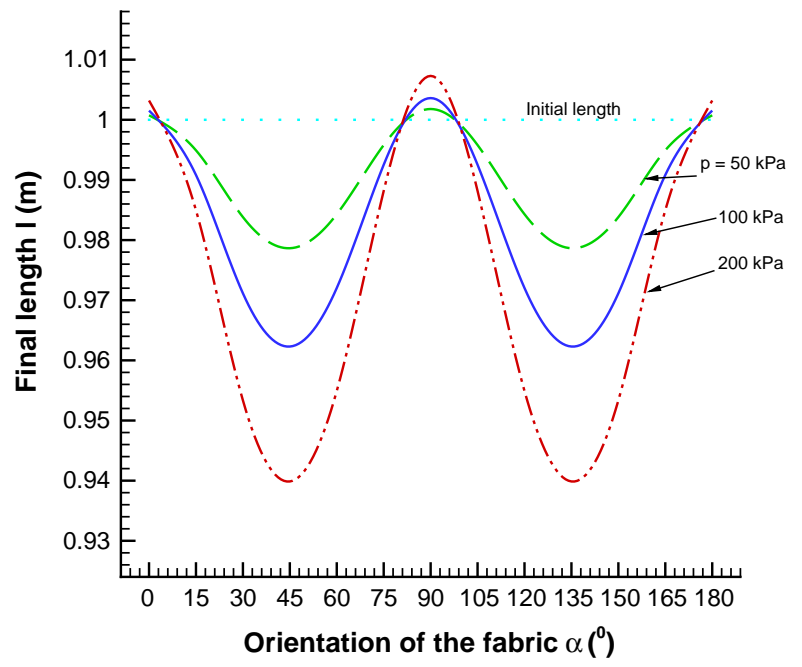

(b) Final length $\ell$

(a) Final radius $a$

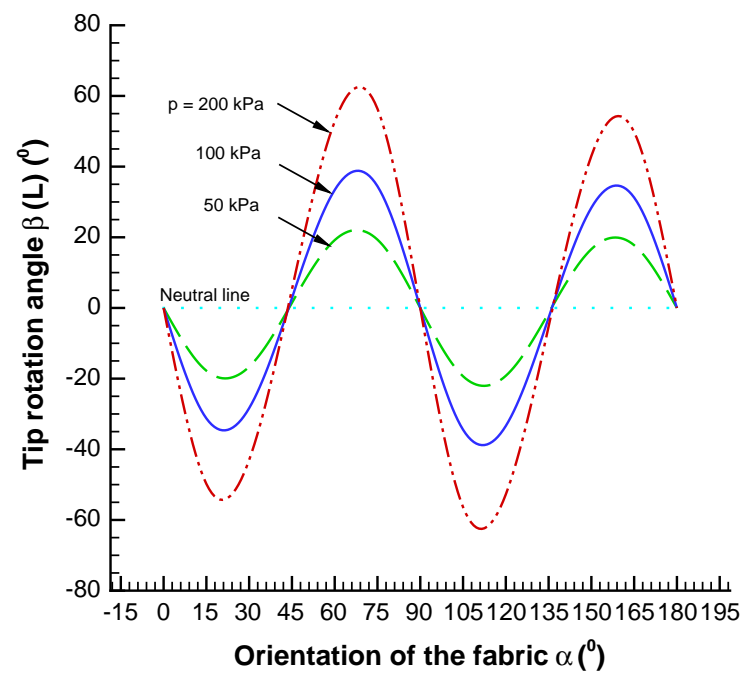

(c) Tip rotation angle $\beta(L)$

Fig. 4. Change of geometry and tip rotation angle vs orientation $\alpha$ of the membrane - Unbalanced material. 


\subsubsection{Unbalanced material}

Fig. 4 obtained for the unbalanced material shows that the graphs of the final radius and length are symmetrical with respect to $\alpha=90^{\circ}$, whereas the graphs of the tip rotation angle are anti symmetrical with respect to $\alpha=90^{\circ}$.

Fig. 4 a shows that for all orientations of the membrane, the fi nal radius $a$ of the tube increases with the internal pressure. At a prescribed value of pressure $p$, the radius changes most when the membrane is oriented at an angle very close to $\alpha=45^{\circ}$ while it changes least when $\alpha=90^{\circ}$. At internal pressure $p=200 \mathrm{kPa}$ for in stance, the final radius reaches its maximal value $a=0.0647 \mathrm{~m} \mathrm{(8 \%}$ increase compared with the initial radius) when $\alpha=44.2^{\circ}$ and its minimal value $a=0.06078 \mathrm{~m}$ ( $1 \%$ increase) when $\alpha=90^{\circ}$. The differ ence between the maximal and the minimal final radii $a_{\max }$ and $a_{\min }$ is more than $6 \%$, it is even larger with increasing internal pressures.

As can be seen in Fig. $4 \mathrm{~b}$, the tube can either lengthen or sur prisingly shorten, depending the orientation of the membrane. At $p=200 \mathrm{kPa}$ for instance, the tube lengthens if $\alpha \in\left[0^{\circ}, 5.9^{\circ}[, \alpha \in] 81.1^{\circ}, 98.9^{\circ}[\right.$ or $\left.\alpha \in] 174.1^{\circ}, 180^{\circ}\right]$, the extremal lengths being $\ell_{\mid \alpha=0^{\circ}}=1.0032 \mathrm{~m}, \ell_{\mid} \alpha=90^{\circ}=1.0073 \mathrm{~m}$. For orientation angle $\alpha$ outside the previous intervals, the tube gets shorter when pressurized, reaching the minimal length of $\ell=0.9399 \mathrm{~m}$ for $\alpha=44.6^{\circ}$. The maximum elongation is $0.73 \%$ for $\alpha=90^{\circ}$, the maxi mal shortening is $6 \%$ for $\alpha=44.6^{\circ}$, the difference between $\ell_{\max }$ and $\ell_{\min }$ is about $7 \%$. There exist four orientations of the membrane that make the length of the inflated tube unchanged: $\alpha=5.9^{\circ}, 81.1^{\circ}$, $98.9^{\circ}$ or $174.1^{\circ}$.

It is noteworthy that the internal pressure does not only change the radius and the length of the tube but it also makes the cross sections rotate about the tube axis, as shown in Fig. 4c. Here, the rotation of the tube is due to the pressure and the fact that the membrane is so oriented that the warp and weft directions do not coincide with the cylindrical ones, not because of an external twisting moment. The cross sections may rotate with positive or negative angles of various magnitudes, depending on the orienta tion of the membrane. At $p=200 \mathrm{kPa}$ for instance, the tip rotation angle of the tube is positive and maximal $\beta(L)=63^{\circ}$ when $\alpha=70^{\circ}$. On the other hand, it is negative and maximal $\beta(L)=63^{\circ}$ when $\alpha=110^{\circ}$, and it is zero when $\alpha=43.2^{\circ}$ or $136.8^{\circ}$ (not to mention the standard angles $\alpha=0^{\circ}, 90^{\circ}$ or $180^{\circ}$ ). In addition, the orientation angles found are not the same accord

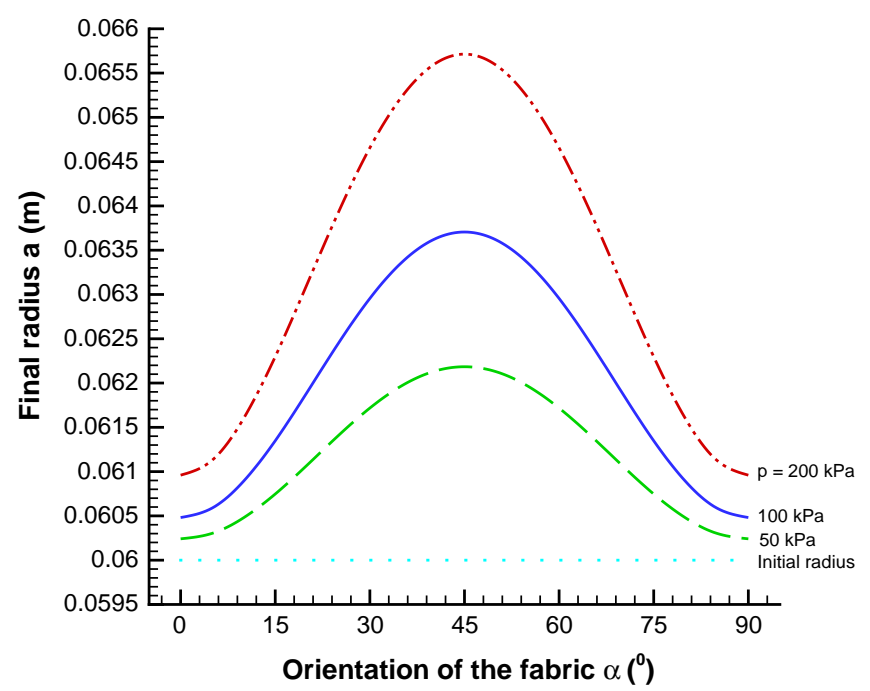

(a) Final radius $a$

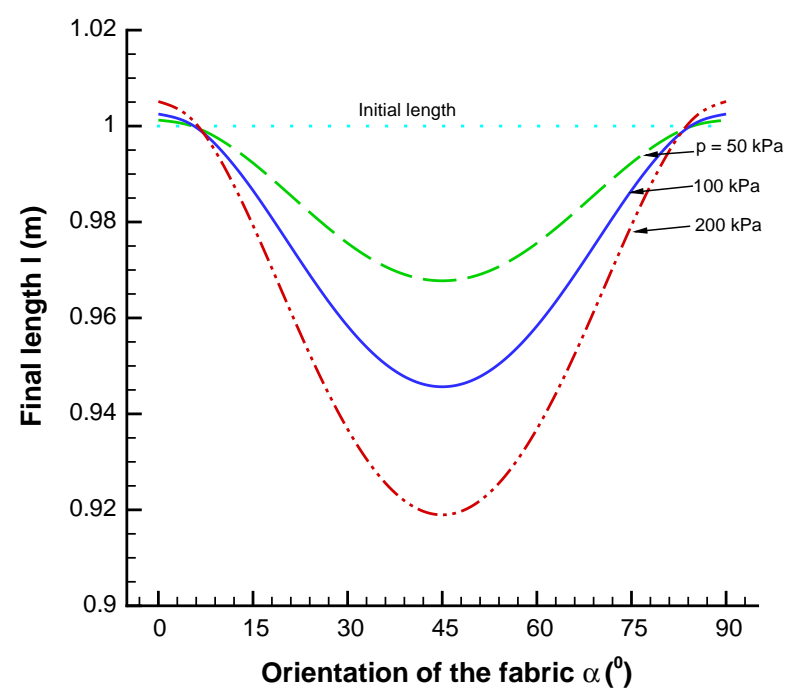

(b) Final length $\ell$

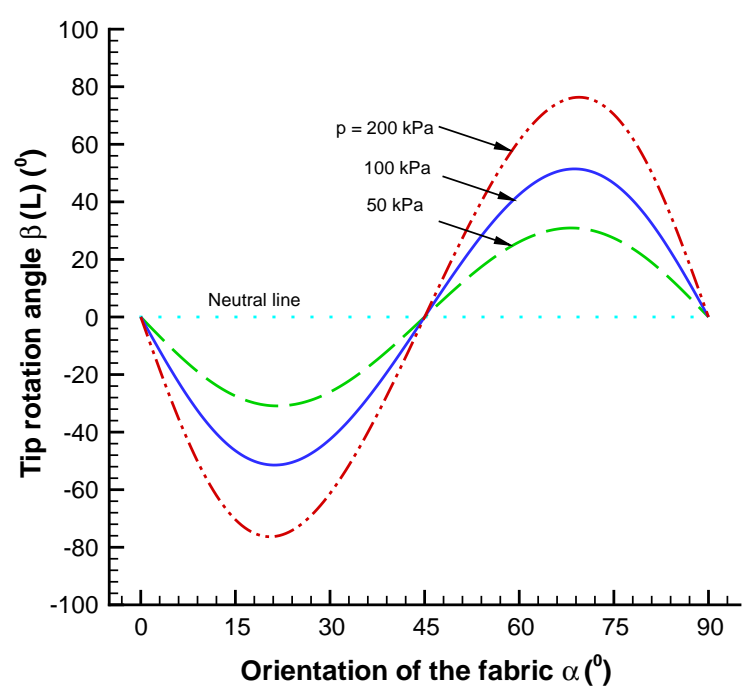

(c) Tip rotation angle $\beta(L)$

Fig. 5. Change of geometry and tip rotation angle vs orientation $\alpha$ of the membrane - Balanced material. 
ing to the prescribed pressure. In the cases when the orthotropy directions of the material coincide with the circumference and the axis of the tube $\left(\alpha=0^{\circ}, 90^{\circ}\right.$ or $\left.180^{\circ}\right)$, the tube does not rotate and this situation has been treated in a previous work [17].

\subsubsection{Balanced material}

Fig. 5 related to the case of the balanced material shows that there are symmetry and antisymmetry with respect to $\alpha=90^{\circ}$ as in the case of the unbalanced material, and also with respect to $\alpha=45^{\circ}$.

The radius of the tube increases most when the membrane is oriented at $\alpha=45^{\circ}$ and least when the warp (resp. weft) direction is parallel to the circumference (resp. the axis) of the tube, i.e. $\alpha=0^{\circ}$ and $\alpha=90^{\circ}$, see Fig. 5a. At $p=200 \mathrm{kPa}$ for instance, the max imal final radius is $a_{\mid \alpha=45^{\circ}}=0.06571 \mathrm{~m}$ (about $10 \%$ increase) and the minimal final radius is $a_{\mid \alpha=0^{\circ}}=a_{\mid \alpha=90^{\circ}}=0.06096 \mathrm{~m}$ (about $2 \%$ in crease). The difference between the maximal and minimal final ra dii $a_{\max }$ and $a_{\min }$ is $7 \%$.

As depicted in Fig. 5b, the final length $\ell$ after inflation has the same features as in the case of unbalanced material. At pressure $p=200 \mathrm{kPa}$, the tube length increases for $\alpha \in\left[0^{\circ}, 6.2^{\circ}[\right.$ and $\alpha \in] 83.8^{\circ}, 90^{\circ}$, and, since the material is balanced, the maximal elongations are equal, $\ell_{\mid} \alpha=0^{\circ}=\ell_{\mid \alpha=90^{\circ}}=1.0051 \mathrm{~m}$ (0.5\% elongation). However, for most of the membrane orientations $\alpha$ $\in] 6.2^{\circ}, 83.8^{\circ}[$ the tube gets shorter when pressurized and the maximal shortening $\ell_{\mid} \alpha=45^{\circ}=0.9189 \mathrm{~m}$ is attained at $\alpha=45^{\circ}$ (more than $8 \%$ shortening). The difference between $\ell_{\max }$ and $\ell_{\min }$ is greater than $9 \%$. Note that the tube length does not change for $\alpha=6.2^{\circ}$ and $83.8^{\circ}$.

As in the case of the balanced material, the orientation angle giving rise to a maximal rotation of the cross sections depends on the prescribed internal pressure. At $p=200 \mathrm{kPa}$ for instance, the tip rotation magnitude $|\beta(L)|$ is maximal if $\alpha=69^{\circ}\left(\beta(L)=74.5^{\circ}\right)$ or $\alpha=21^{\circ}\left(\beta(L)=74.5^{\circ}\right)$, see Fig. $5 c$. On the other hand, in contrast to the case of the unbalanced material, here the inflated tube does not rotate not only when the membrane is oriented at standard angles $\alpha=0^{\circ}$ and $90^{\circ}$, but also when $\alpha=45^{\circ}$, and this holds whatever the internal pressure.

\subsection{Change of geometry and rotation versus the internal pressure}

The final radius $a$, the final length $\ell$ and the tip rotation angle $\beta(L)$ are plotted in Figs. 6 and 7 versus the internal pressure $p$

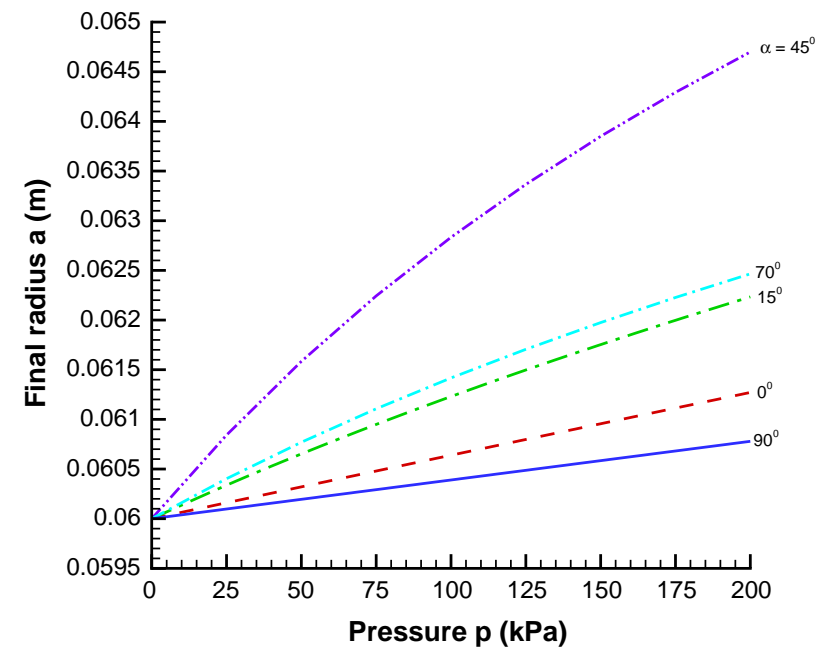

(a) Final radius $a$

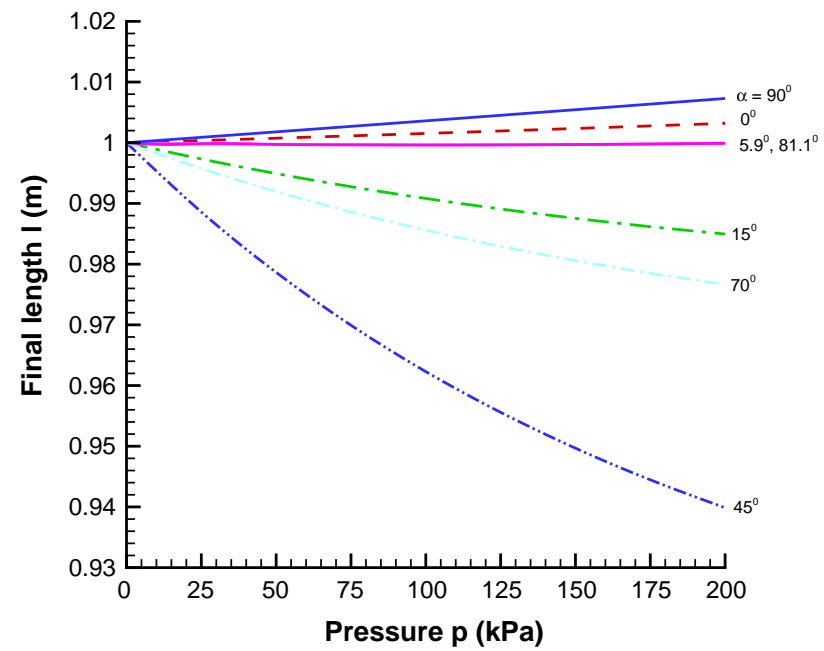

(b) Final length $\ell$

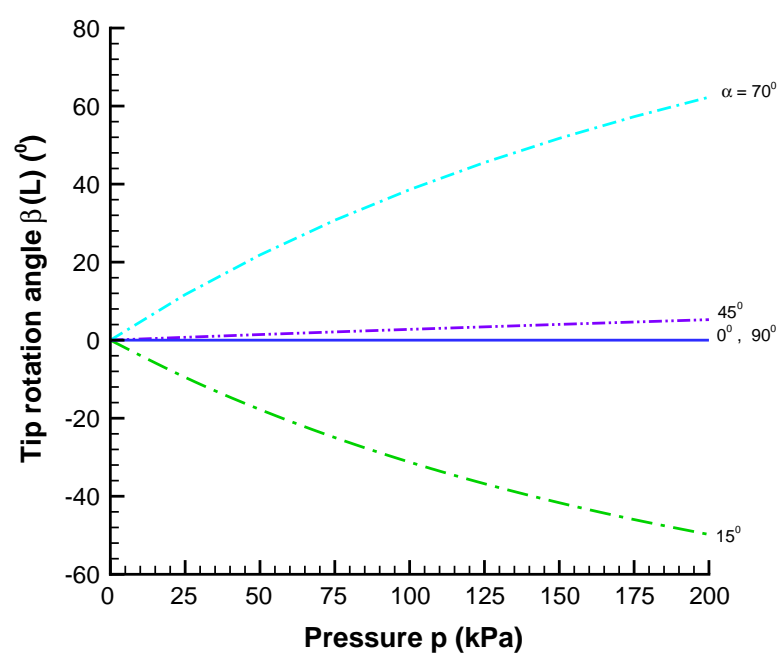

(c) Tip rotation angle $\beta(L)$

Fig. 6. Change of geometry and tip rotation vs internal pressure $p$ - Unbalanced material 
ranging from 0 to $200 \mathrm{kPa}$. For $\alpha=45^{\circ}$, these quantities vary as nonlinear functions of $p$ whereas for $\alpha=0^{\circ}$ or $90^{\circ}$, they vary al most linearly. In fact, numerical computations show that, under larger internal pressures, all the considered quantities are non linear functions, for all orientations of the membrane. However, the numerical results are here presented for $p$ smaller than $200 \mathrm{kPa}$ only, so as to limit the tensile stresses in the membrane.

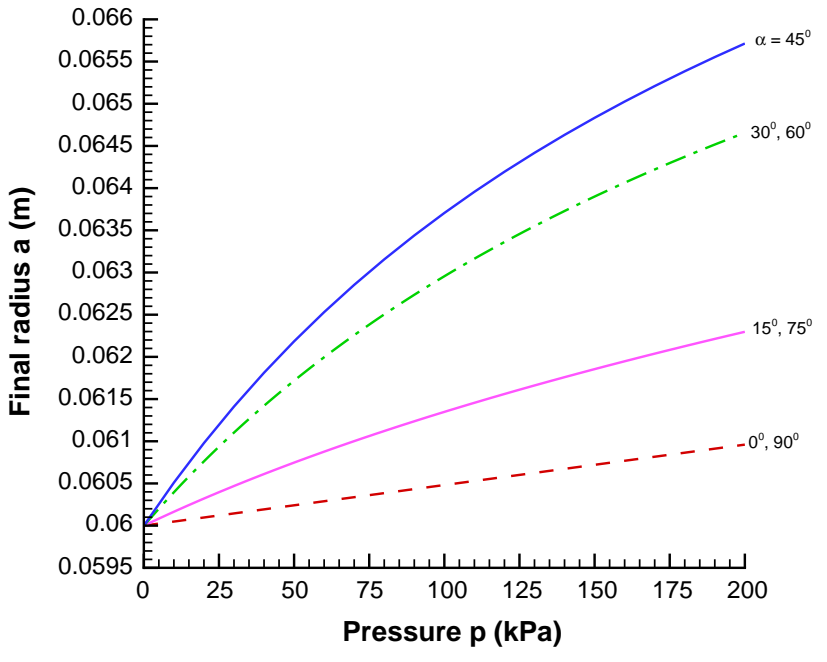

(a) Final radius $a$

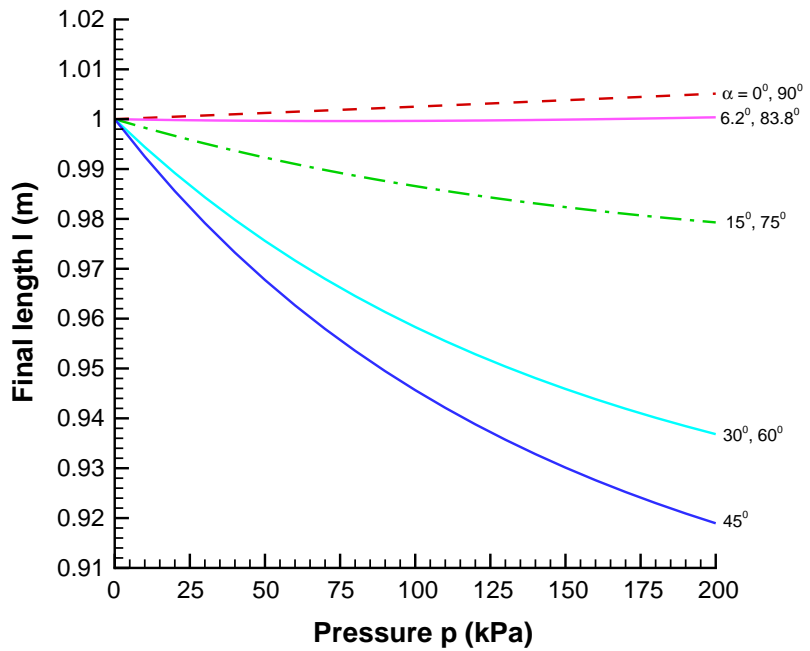

(b) Final length $\ell$

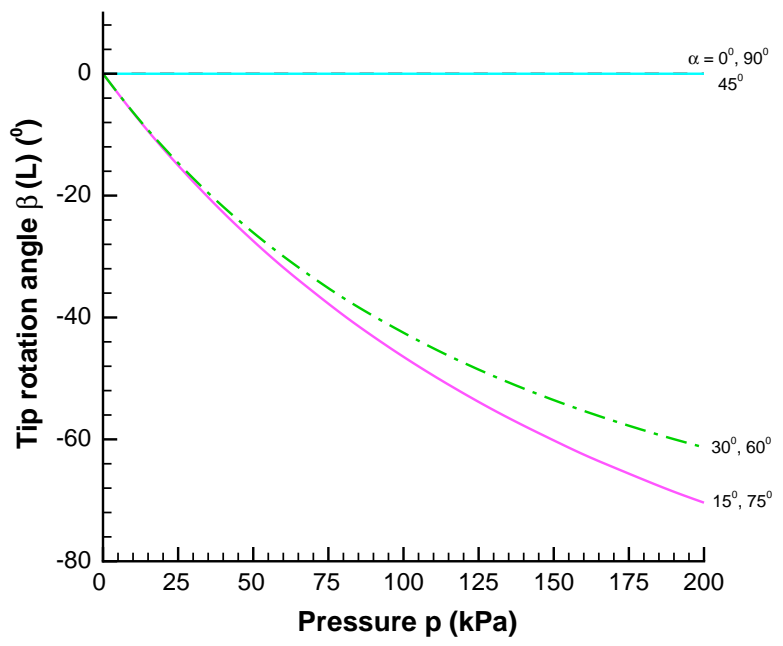

(c) Tip rotation angle $\beta(L)$

Fig. 7. Change of geometry and tip rotation vs internal pressure $p$ - Balanced material.

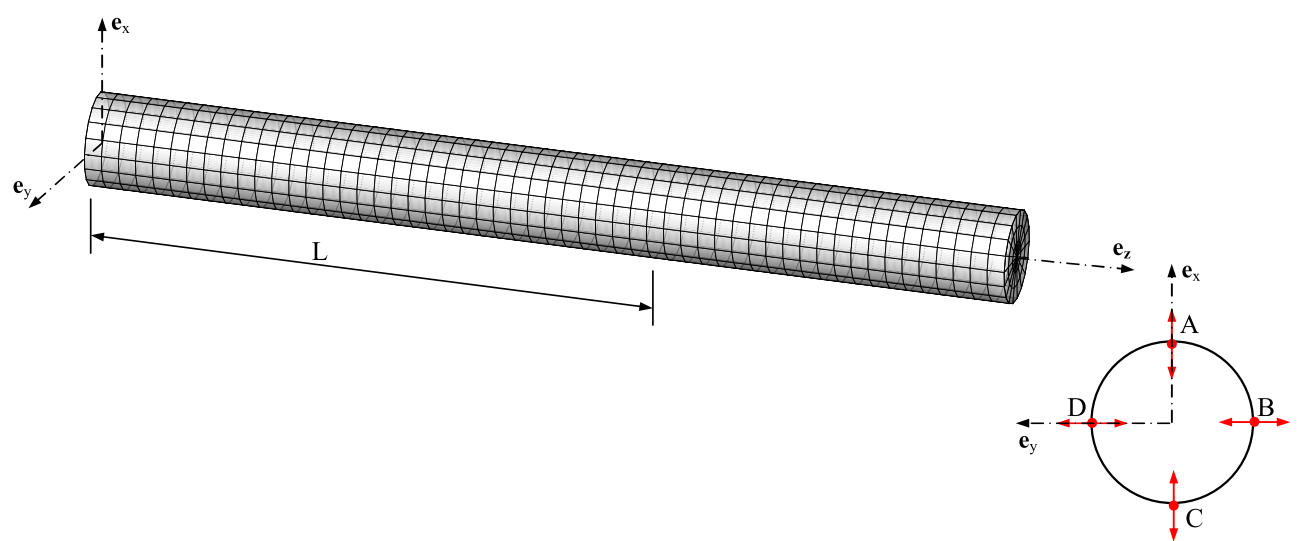

Fig. 8. (a) Mesh of the tube with membrane finite elements. (b) Points $A, B, C, D$ in section $Z=0$ and allowed directions of motion. 


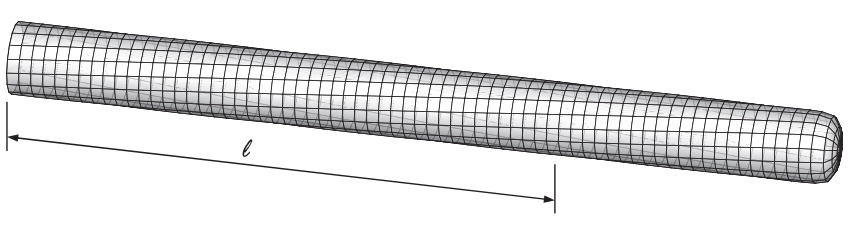

Fig. 9. Deformed configuration of the tube.

\section{Finite element validation}

In order to evaluate the proposed theory, let us compare the solution of the nonlinear system (22) with that obtained from a fi nite element code. Here, use is made of an in house multi purpose computer program developed for nonlinear structural mechanics analysis.

The tube is modeled as a 3D membrane structure, with the membrane finite elements having no bending stiffness and satisfy ing the plane stress condition. In section $Z=0$, the axial displace ment of all the nodes is set to zero while the displacements in plane $(X Y)$ of nodes $(A, B, C, D)$ shown in Fig. 8 are appropriately pre scribed in order to preserve the symmetries and preclude all rigid body motions. The numerical data for the geometries and the elas tic properties are those given in Table 1 for the unbalanced and bal anced materials.

The total Lagrangian weak form of the problem is discretized by means of the finite element method and results in a nonlinear sys tem of algebraic equations which can be solved by the iterative Newton's scheme. The tangent stiffness matrix is the sum of the standard stiffness due to internal stresses and the stiffness due to the pressure which is a follower force. The internal pressure $p$ is applied progressively. In the first step when $p$ is equal to zero, the stiffness matrix is singular as is the case for any non pre stressed membrane structure and it is necessary to add a small fic titious prestress to achieve the convergence. Of course, this prestress is immediately canceled in the subsequent computa tional steps.

The theoretical Eq. (22) have been established assuming that the deformed tube remains cylindrical. Here, in numerical compu tations the tube end is modeled as a cap made of an isotropic mem brane just to close up the tube, and the deformed tube is not quite cylindrical because of this cap. For this reason, one has to consider a tube which is longer than the theoretical length $L$, as shown in

Table 2

Comparison between the solution of (22) and the membrane finite element solution

\begin{tabular}{|c|c|c|c|c|c|c|c|c|c|c|}
\hline \multirow[t]{2}{*}{$\alpha$} & \multirow[t]{2}{*}{$p(\mathrm{kPa})$} & \multicolumn{3}{|c|}{ Solution of (22) } & \multicolumn{3}{|c|}{ Membrane finite element solution } & \multicolumn{3}{|c|}{ Difference (\%) } \\
\hline & & $a(\mathrm{~m})$ & $\ell(\mathrm{m})$ & $\beta(L)\left(^{\circ}\right)$ & $a(\mathrm{~m})$ & $\ell(\mathrm{m})$ & $\beta(L)\left(^{\circ}\right)$ & in $a$ & in $\ell$ & in $\beta(L)$ \\
\hline \multicolumn{11}{|c|}{ Balanced material: $A=0.06 \mathrm{~m}, L=1 \mathrm{~m}, H=6 \times 10^{4} \mathrm{~m}, E_{\ell} H=E_{t} H=660 \mathrm{kN} / \mathrm{m}, v_{\ell t}=v_{t \ell}=0.23, G_{\ell t}=9 \mathrm{kN} / \mathrm{m}$} \\
\hline \multirow[t]{7}{*}{$0^{\circ}$} & 50 & 0.06024 & 1.00124 & 0 & 0.06024 & 1.00124 & $3.26 \mathrm{E}-14$ & $<0.01$ & $<0.01$ & /I \\
\hline & 75 & 0.06036 & 1.00187 & 0 & 0.06036 & 1.00187 & $-4.40 \mathrm{E}-15$ & $<0.01$ & $<0.01$ & $\|$ \\
\hline & 100 & 0.06048 & 1.00251 & 0 & 0.06048 & 1.00251 & $-7.04 \mathrm{E}-14$ & $<0.01$ & $<0.01$ & /l \\
\hline & 125 & 0.06060 & 1.00315 & 0 & 0.06060 & 1.00315 & $1.08 \mathrm{E}-14$ & $<0.01$ & $<0.01$ & /I \\
\hline & 150 & 0.06072 & 1.00380 & 0 & 0.06072 & 1.00380 & $-3.40 \mathrm{E}-13$ & $<0.01$ & $<0.01$ & /I \\
\hline & 175 & 0.06084 & 1.00445 & 0 & 0.06084 & 1.00445 & $4.12 \mathrm{E}-13$ & $<0.01$ & $<0.01$ & /l \\
\hline & 200 & 0.06096 & 1.00512 & 0 & 0.06096 & 1.00512 & $-8.62 \mathrm{E}-14$ & $<0.01$ & $<0.01$ & $1 /$ \\
\hline \multirow[t]{7}{*}{$30^{\circ}$} & 50 & 0.06172 & 0.97559 & -26.0762 & 0.06172 & 0.97559 & -26.0793 & $<0.01$ & $<0.01$ & $<0.05$ \\
\hline & 75 & 0.06238 & 0.96622 & -35.1667 & 0.06238 & 0.96622 & -35.1704 & $<0.01$ & $<0.01$ & $<0.05$ \\
\hline & 100 & 0.06296 & 0.95831 & -42.5177 & 0.06295 & 0.95831 & -42.5226 & $<0.01$ & $<0.01$ & $<0.05$ \\
\hline & 125 & 0.06346 & 0.95160 & -48.5479 & 0.06346 & 0.95160 & -48.5530 & $<0.01$ & $<0.01$ & $<0.05$ \\
\hline & 150 & 0.06390 & 0.94589 & -53.5560 & 0.06390 & 0.94589 & -53.5671 & $<0.01$ & $<0.01$ & $<0.05$ \\
\hline & 175 & 0.06430 & 0.94101 & -57.7604 & 0.06430 & 0.94101 & -57.7724 & $<0.01$ & $<0.01$ & $<0.05$ \\
\hline & 200 & 0.06466 & 0.93683 & -61.3232 & 0.06466 & 0.93682 & -61.3245 & $<0.01$ & $<0.01$ & $<0.05$ \\
\hline \multirow[t]{7}{*}{$45^{\circ}$} & 50 & 0.06218 & 0.96775 & 0 & 0.06218 & 0.96774 & $-1.25 \mathrm{E}-14$ & $<0.01$ & $<0.01$ & /I \\
\hline & 75 & 0.06301 & 0.95570 & 0 & 0.06301 & 0.95569 & $2.35 \mathrm{E}-14$ & $<0.01$ & $<0.01$ & /I \\
\hline & 100 & 0.06371 & 0.94565 & 0 & 0.06370 & 0.94565 & $-4.56 \mathrm{E}-15$ & $<0.01$ & $<0.01$ & /I \\
\hline & 125 & 0.06431 & 0.93723 & 0 & 0.06431 & 0.93722 & $-9.15 \mathrm{E}-14$ & $<0.01$ & $<0.01$ & /I \\
\hline & 150 & 0.06483 & 0.93012 & 0 & 0.06483 & 0.93012 & $1.59 \mathrm{E}-14$ & $<0.01$ & $<0.01$ & /I \\
\hline & 175 & 0.06529 & 0.92409 & 0 & 0.06529 & 0.92409 & $3.74 \mathrm{E}-14$ & $<0.01$ & $<0.01$ & /I \\
\hline & 200 & 0.06571 & 0.91895 & 0 & 0.06571 & 0.91895 & $-2.69 \mathrm{E}-13$ & $<0.01$ & $<0.01$ & /I \\
\hline \multicolumn{11}{|c|}{ Unbalanced material: $A=0.06 \mathrm{~m}, L=1 \mathrm{~m}, H=6 \times 10^{4} \mathrm{~m}, E_{\ell}=780 \mathrm{kN} / \mathrm{m}, E_{t}=504 \mathrm{kN} / \mathrm{m}, v_{\ell t}=0.31, v_{t \ell}=0.2, G_{\ell t}=13.8 \mathrm{kN} / \mathrm{m}$} \\
\hline \multirow[t]{7}{*}{$0^{\circ}$} & 50 & 0.06032 & 1.00075 & 0 & 0.06032 & 1.00075 & $7.64-14$ & $<0.01$ & $<0.01$ & /I \\
\hline & 75 & 0.06048 & 1.00114 & 0 & 0.06048 & 1.00114 & $-4.15 \mathrm{E}-14$ & $<0.01$ & $<0.01$ & /l \\
\hline & 100 & 0.06064 & 1.00153 & 0 & 0.06064 & 1.00153 & $-1.23 \mathrm{E}-15$ & $<0.01$ & $<0.01$ & /I \\
\hline & 125 & 0.06080 & 1.00194 & 0 & 0.06080 & 1.00194 & $2.04 \mathrm{E}-14$ & $<0.01$ & $<0.01$ & /I \\
\hline & 150 & 0.06096 & 1.00235 & 0 & 0.06096 & 1.00235 & $-3.47 \mathrm{E}-14$ & $<0.01$ & $<0.01$ & /I \\
\hline & 175 & 0.06111 & 1.00278 & 0 & 0.06111 & 1.00278 & $1.25 \mathrm{E}-15$ & $<0.01$ & $<0.01$ & /I \\
\hline & 200 & 0.06127 & 1.00321 & 0 & 0.06127 & 1.00321 & $-1.52 \mathrm{E}-13$ & $<0.01$ & $<0.01$ & II \\
\hline \multirow[t]{7}{*}{$30^{\circ}$} & 50 & 0.06129 & 0.98382 & -16.6429 & 0.06129 & 0.98382 & -16.6502 & $<0.01$ & $<0.01$ & $<0.05$ \\
\hline & 75 & 0.06184 & 0.97710 & -22.9962 & 0.06184 & 0.97710 & -23.0164 & $<0.01$ & $<0.01$ & $<0.05$ \\
\hline & 100 & 0.06234 & 0.97115 & -28.3647 & 0.06234 & 0.97115 & -28.3741 & $<0.01$ & $<0.01$ & $<0.05$ \\
\hline & 125 & 0.06280 & 0.96587 & -32.9260 & 0.06280 & 0.96587 & -32.9347 & $<0.01$ & $<0.01$ & $<0.05$ \\
\hline & 150 & 0.06322 & 0.96119 & -36.8194 & 0.06322 & 0.96119 & -36.8204 & $<0.01$ & $<0.01$ & $<0.05$ \\
\hline & 175 & 0.06361 & 0.95703 & -40.1554 & 0.06361 & 0.95703 & -40.1569 & $<0.01$ & $<0.01$ & $<0.05$ \\
\hline & 200 & 0.06397 & 0.95335 & -43.0229 & 0.06397 & 0.95334 & -43.0237 & $<0.01$ & $<0.01$ & $<0.05$ \\
\hline \multirow[t]{7}{*}{$45^{\circ}$} & 50 & 0.0615 & 0.97865 & 1.4390 & 0.06158 & 0.97865 & 1.4392 & $<0.01$ & $<0.01$ & $<0.01$ \\
\hline & 75 & 0.06224 & 0.96994 & 2.1172 & 0.06224 & 0.96993 & 2.1170 & $<0.01$ & $<0.01$ & $<0.01$ \\
\hline & 100 & 0.06283 & 0.96230 & 2.7747 & 0.06283 & 0.96230 & 2.7748 & $<0.01$ & $<0.01$ & $<0.01$ \\
\hline & 125 & 0.06337 & 0.95559 & 3.4150 & 0.06337 & 0.95559 & 3.4154 & $<0.01$ & $<0.01$ & $<0.01$ \\
\hline & 150 & 0.06385 & 0.94968 & 4.0407 & 0.06385 & 0.94968 & 4.0410 & $<0.01$ & $<0.01$ & $<0.01$ \\
\hline & 175 & 0.06430 & 0.94447 & 4.6536 & 0.06430 & 0.94447 & 4.6529 & $<0.01$ & $<0.01$ & $<0.01$ \\
\hline & 200 & 0.06470 & 0.93987 & 5.2552 & 0.06470 & 0.93986 & 5.2557 & $<0.01$ & $<0.01$ & $<0.01$ \\
\hline
\end{tabular}


Fig. 8. If the extra length is large enough, the portion of the tube between $Z=0$ and $Z=L$ remains quite cylindrical.

The mesh contains 3217 nodes and 1072 membrane elements (see Fig. 8), which proves to be sufficient for all numerical calcula tions since in the case of the unbalanced material submitted an internal pressure $p=200 \mathrm{kPa}$ for instance, the maximum difference with a finer mesh containing 6643 nodes is only $0.02 \%$.

Fig. 9 shows a typical deformed shape of the tube when the membrane orientation $\alpha$ is different from zero. As in Fig. 8, the lines in Fig. 9 represent the finite element mesh, not the orthotropy directions of the membrane. The deformed mesh clearly shows that the tube undergoes a rotation $\beta(Z)$ around its axis due to the combined effect of the membrane orientation $\alpha \neq 0$ and the pre scribed pressure. It should be noted that the rotation takes place without any external twisting torque.

Table 2 presents the solutions of the nonlinear system (22) compared with the finite element results obtained for the unbal anced and balanced materials. For each material, the computations are undertaken considering three orientations of the membrane, $\alpha=0^{\circ}, 30^{\circ}$ and $45^{\circ}$, and pressures ranging from 50 to $200 \mathrm{kPa}$ by steps of $25 \mathrm{kPa}$. The final length $\ell$ in Fig. 9 corresponds to the initial length $L$ in Fig. 8, and the tip rotation $\beta(L)$ is the rotation of the cross section $Z=L$ in Fig. 8. The solution of (22) and the finite ele ment solution are found to be in very good accordance since the difference between them is less than $0.05 \%$ in all cases presented and it is also of the same range in other cases not presented here.

\section{Conclusions}

This paper deals with the inflation of an orthotropic membrane tube whose warp or weft directions are not necessarily parallel to the tube axis. The problem has been formulated in finite deforma tions so as to take account of the finite rotations of the tube, and it has been shown that the theoretical solution can be obtained by solving a system of three algebraic equations. The numerical com putations have been carried out on two types of material, giving the final length, radius and the rotation of the tube as nonlinear functions of the inflating pressure. Also, they clearly show the sig nificant influence of the orientation of the membrane on the final shape of the inflated tube. Eventually, it has been shown that the proposed theoretical solution gives results which are in very good accordance with those obtained from an independent membrane finite element code.

Investigations are in progress making use of the obtained ana lytical solution and by the inverse analysis in order to identify the elastic properties of orthotropic membranes.

\section{References}

[1] Comer RL, Levy S. Deflections of an inflated circular cylindrical cantilever beam. AIAA J 1963;1(7):1652-5.

[2] Douglass WJ. Bending stiffness of an inflated cylindrical cantilever beam. AIAA J 1969;7:1248-53.

[3] Webber JPH. Deflections of inflated cylindrical cantilever beams subjected to bending and torsion. Aeronaut J 1982;1020:306-12.

[4] A Main J, W Peterson S, Strauss AM. Load-deflection behaviour of space-based inflatable fabric beams. J Aerospace Eng 1994;7(2):225-38.

[5] Main JA, Peterson SW, Strauss AM. Beam-type bending of space-based membrane structures. J Aerospace Eng 1995;8(2):120-5.

[6] Steeves EC. A linear analysis of the deformation of pressure stabilized beams. Technical Report 75-47-AMEL, US Army Natick Laboratories; 1975.

[7] Fichter WB. A theory for inflated thin-wall cylindrical beams. NASA Technical Note, NASA TND-3466; 1966.

[8] Wielgosz C, Thomas JC. Deflection of inflatable fabric panels at high pressure. Thin-Walled Struct 2002;40:523-36.

[9] Wielgosz C, Thomas JC. Deflections of highly inflated fabric tubes. Thin-Walled Struct 2004;42:1049-66.

[10] Le van A, Wielgosz C. Bending and buckling of inflatable beams: some new theoretical results. Thin-Walled Struct 2005;43:1166-87.

[11] Apedo KL, Ronel S, Jacquelin E, Massenzio M, Bennani A. Theoretical analysis of inflatable beams made from orthotropic fabric. Thin-Walled Struct 2009;47:1507-22.

[12] Nguyen TT, Ronel S, Massenzio M, Apedo KL, Jacquelin E. Analytical buckling analysis of an inflatable beam made of orthotropic technical textiles. ThinWalled Struct 2012;51:186-200.

[13] Nguyen QT, Thomas JC, Le van A. Some new theoretical results for the orthotropic inflatable beams. In Proceedings of the ASME 2012 11th biennal conference on engineering systems design and analysis (ESDA 2012), July 2nd4th, Nantes, France; 2012.

[14] Pargana JB, Lloyd Smith D, Izzudin BA. Advanced material model for coated fabrics used in tensionned fabric structures. Eng Struct 2007;29:1323-36.

[15] Cavallaro PV, Jonhson ME, Sadegh AM. Mechanics of plain-woven fabrics for inflated structures. Compos Struct 2003;61:375-93.

[16] Galliot C, Luchsinger R. A simple model describing the non-linear biaxial tensile behaviour of PVC-coated polyester fabrics for use in finite element analysis. Compos Struct 2009;90:437-47.

[17] Thomas JC, Le van A. An exact solution for inflated orthotropic membrane 637 tubes. Thin-walled Structure 2013;61:116-20. 